\title{
Cutaneous Microvascular Dysfunction Is Associated With Human Leukocyte Antigen-DQ in Youths With Type 1 Diabetes
}

\author{
MICHAL ODERMARSKY, ÅKE LERNMARK, LENNART TRUEDSSON, AND PETRU LIUBA \\ Pediatric Cardiology [M.O., P.L.], Lund University Hospital, Lund, Sweden 221 85; Diabetes and Celiac Unit [A.L.], Lund University \\ Hospital, Malmö, Sweden 205 02; Clinical Microbiology and Immunology [L.T.], Lund University Hospital, Lund, Sweden 22185
}

\begin{abstract}
Functional disturbances in microcirculation in juvenile type 1 diabetes (T1D) are believed to underlie, in part, the later occurrence of cardiovascular complications. Some epidemiologic studies suggested greater risk of microvascular complications in those with T1D-risk genotypes of human leukocyte antigen (HLA). We investigated whether HLA-DQ2/8, which is linked to highest T1D morbidity, influences microvascular function in young diabetic patients. Cutaneous microvascular endothelium-dependent and independent reactivity and HLA genotypes were assessed in young patients (age: 9-21 y) with T1D (duration: 2-20 y). HLA-DQ2/8 was identified in 29 of 75 patients. The DQ2/8 and non-DQ2/8 groups were similar in age, body mass index, diabetes duration, glycosylated hemoglobin, and C-reactive protein (CRP). Compared with the nonDQ2/8 group, the DQ2/8 group showed decreased endotheliumdependent responses $(p=0.03$ after adjustment for age, diabetes duration, glycosylated hemoglobin, and CRP) and elevated soluble intercellular adhesion molecule-1 $(p=0.05)$. In these but not in non-DQ2/8 patients, CRP correlated with both systolic $(r=0.76$; $p<0.001)$ and diastolic $(r=0.50 ; p=0.01)$ blood pressure. HLA-DQ2/8 is associated with endothelial microvascular dysfunction in young patients with T1D, and future studies are needed to provide mechanistic insights. The findings could explain in part the previously reported epidemiologic link between T1D-risk HLA and microvascular complications. (Pediatr Res 63: 420-422, 2008)
\end{abstract}

A broad spectrum of cardiovascular risk factors including type 1 diabetes (T1D) has been linked to functional disturbances in microcirculation. The generalized microvascular dysfunction in T1D is an important mechanism in the development of microvascular diabetes complications in, for instance, eyes, kidneys, or myocardium (1). Although the precise pathophysiology of diabetes microvasculopathy remains elusive, it seems that both extrinsic (e.g., infection, socioeconomic status) and intrinsic (race, ethnicity, metabolic control) factors contribute to it, in part, through progressive injury to microvascular endothelial cells (2).

Some, but not all, previous epidemiologic works suggested a possible link between the human leukocyte antigen (HLA) system and clinical manifestations of microvasculopathy in both diabetic and nondiabetic patients $(3,4)$. Expression of diabetes-risk HLA class II molecules on islet endothelial cells could

Received September 26, 2007; accepted December 16, 2007.

Correspondence: Petru Liuba, Ph.D., M.D., Division of Pediatric Cardiology, Department of Pediatrics, Lund University Hospital, 22185 Lund, Sweden; e-mail: petru.liuba@med.lu.se

The study was supported by FAMRI (PL) and Lund University Hospital (PL).

DOI: 10.1203/PDR.0b013e318165bfd4 be a central vascular event in the pathogenesis of T1D (5). Previous studies showing the ability of endothelial cells in other vascular beds to express HLA class II support the assumption that HLA may be present in the entire vascular system $(6,7)$. Whether diabetes-risk HLA is associated with systemic microvascular endothelial dysfunction has not yet been studied.

Herein, we investigated the relationship between the HLADQ2/8 genotype, which confers the highest risk for T1D (8) and endothelial function of cutaneous microcirculation in young patients with T1D.

\section{METHODS}

Seventy-five children and adolescents (45 male and 30 female, mean age: $16 \mathrm{y}$, range: $9-21 \mathrm{y}$ ) with T1D (mean: $8 \mathrm{y}$, range: $2-20 \mathrm{y}$ ) were randomly recruited from the pediatric outpatient diabetes clinic at the Lund University Hospital. Exclusion criteria were familiar hypercholesterolemia, infectious illness, active smoking, history of premature coronary or cerebrovascular disease among first-degree relatives, and systemic hypertension. Results of ocular examination were gathered from the patients' medical records.

HLA genotypes were determined in dried spots of peripheral blood by polymerase chain reaction followed by DELFIA hybridization assay (9). Plasma high-sensitivity C-reactive protein (CRP) was measured by enzymelinked immunoassay using polyclonal antibodies (DACO Diagnostics, Glostrup, Denmark). Plasma levels of soluble intercellular adhesion molecule (sICAM)-1 were measured by an ELISA method (R\&D Systems, Minneapolis, MN).

Blood pressure was measured in the left arm using a Dinamap device. Cutaneous blood flow responses to endothelium-dependent and independent agonists were assessed by two observers in 54 patients using a laser Doppler multifiber probe (481-1; Perimed AB, Sweden) during transdermal iontophoresis of acetylcholine (Ach) and sodium nitroprusside (SNP), respectively, on the volar side of the forearm. The nondominant upper extremity was chosen in all patients. Anodal iontophoresis was used for Ach, whereas SNP was delivered via cathodal iontophoresis. The current was set at $100 \mu \mathrm{A}$ for $20 \mathrm{~s}$ for both drugs, based on previous work (10). Five consecutive doses were applied for both drugs to generate dose-response curves. Baseline perfusion and changes in response to Ach were expressed as area under the curve.

At the time of scanning, both observers were unaware of patients' metabolic and genetic characteristics. In a previous reproducibility study of 10 patients, the intraobserver and interobserver coefficients of variation for skin responses to Ach were $10.4 \%$ and $7.5 \%$, respectively.

The study was approved by the ethical committee for human research at the Lund University. Written informed consent was obtained from all participants over $18 \mathrm{y}$ or from their guardians if under $18 \mathrm{y}$ of age. All participants gave oral consent.

Statistical analyses. The differences between HLA groups in dosedependent responses to Ach and SNP were compared using two-way ANOVA for repeated measurements. ANCOVA was used to control the associations of HLA-DQ2/8 with microvascular responses for the possible confounding

Abbreviations: Ach, acetylcholine; CRP, C-reactive protein; HLA, human histocompatibility leukocyte antigen; $\mathbf{H b A}_{\mathbf{1}}$, glycosylatedhemoglobin; sICAM-1, soluble intercellular adhesion molecule-1; SNP, sodium nitroprusside; T1D, type 1 diabetes 
effects of age, diabetes duration, CRP, and glycosylated hemoglobin $\left(\mathrm{HbA}_{1 \mathrm{C}}\right)$. Due to its skewed distribution, CRP was log-transformed and used as such in all analyses. $p$ less than or equal to 0.05 was considered of statistical significance. Data are mean $\pm \mathrm{SD}$, unless otherwise specified. All analyses were performed using StatView 5.0 software (SAS Institute Inc., Cary, NC).

\section{RESULTS}

HLA-DQ2/8 was identified in 29 patients whereas the remaining 46 patients were negative for this genotype. Both groups were similar with respect to age $(p=0.28)$, body mass index $(p=0.58)$, diabetes duration $(p=0.18), \operatorname{HbA}_{1 \mathrm{c}}(p=$ $0.18)$, CRP $(p=0.22)$, systolic $(p=0.81)$, and diastolic $(p=$ $0.52)$ blood pressure. sICAM-1 was elevated in the DQ2/8 group compared with the non-DQ2/8 group $(p=0.05)$. Incipient (background) retinopathy was detected in 10 of 62 patients who underwent routine ocular examination. No difference in the prevalence of retinopathy was observed between the groups. Patients' clinical and biochemical characteristics are summarized in Table 1.

Although the baseline skin perfusion values were similar $(p=0.29)$, the microvascular responses to Ach were decreased in DQ2/8 group compared with the non-DQ2/8 group $(p=0.01$; Fig. 1/Panel $A)$. The difference remained significant after adjustment for age, diabetes duration, $\mathrm{HbA}_{1 \mathrm{c}}$, and body mass index (ANCOVA, $p=0.03$ ). In contrast, no significant difference in the responses to SNP was noted between the groups ( $p=0.16$; data not shown).

Among the DQ2/8 patients, CRP showed significant correlation with both systolic $(r=0.76 ; p<0.001)$ and diastolic $(r=0.50 ; p=0.01)$ blood pressure (Fig. 1/Panel B). No such association was observed in the non-DQ2/8 group $(r<0.2$ and $p>0.2$ for both; Fig. 1/Panel B). No other correlations related to this genotype were observed.

\section{DISCUSSION}

Laser Doppler iontophoresis emerges as a valid method to study the relationship between cardiovascular risk factors and microvascular function (10). By employing this technique, the

Table 1. Patients' clinical and biochemical characteristics based on HLA-DQ 2/8 genotype

\begin{tabular}{lcc}
\hline & DQ2/8 & non-DQ2/8 \\
\hline Number of patients $(n)$ & 29 & 46 \\
Gender $(\mathrm{M} / \mathrm{F})$ & $16 / 13$ & $29 / 17$ \\
Age $(\mathrm{y})$ & $16 \pm 3$ & $17 \pm 3$ \\
Diabetes duration $(\mathrm{y})$ & $7 \pm 4$ & $9 \pm 4$ \\
$\quad>5 \mathrm{y}(\mathrm{n} / \%)$ & $21 / 72$ & $36 / 78$ \\
$\mathrm{BMI}\left(\mathrm{kg} / \mathrm{m}^{2}\right)$ & $22.4 \pm 4.6$ & $21.9 \pm 3.3$ \\
$\mathrm{SBP}(\mathrm{mm} \mathrm{Hg})$ & $113 \pm 11$ & $112 \pm 11$ \\
$\mathrm{DBP}(\mathrm{mm} \mathrm{Hg})$ & $72 \pm 8$ & $71 \pm 8$ \\
$\mathrm{HbA}(\%)$ & $7.6 \pm 1.6$ & $7.2 \pm 1.2$ \\
$\geq 9 \%(n / \%)$ & $3 / 10$ & $5 / 11$ \\
$\mathrm{CRP}(\mathrm{mg} / \mathrm{L})$ & $1.0 \pm 1.0$ & $1.5 \pm 1.6$ \\
sICAM-1 $(\mu \mathrm{g} / \mathrm{L})$ & $283 \pm 54 *$ & $258 \pm 43$ \\
Patients screened for retinopathy $(n)$ & 24 & 38 \\
Confirmed retinopathy $(n / \%)$ & $4 / 17$ & $6 / 16$ \\
\hline
\end{tabular}

M, males; F, females; BMI, body mass index; SBP, systolic blood pressure; DBP, diastolic blood pressure; $\mathrm{HbA}_{1 \mathrm{c}}$, glycosylated hemoglobin; sICAM-1, soluble intracellular adhesion molecule $1 ; n$ denotes number of patients; * $p=$ $0.05 v s$ the non-DQ 2/8 group. Data are expressed as mean \pm SD.
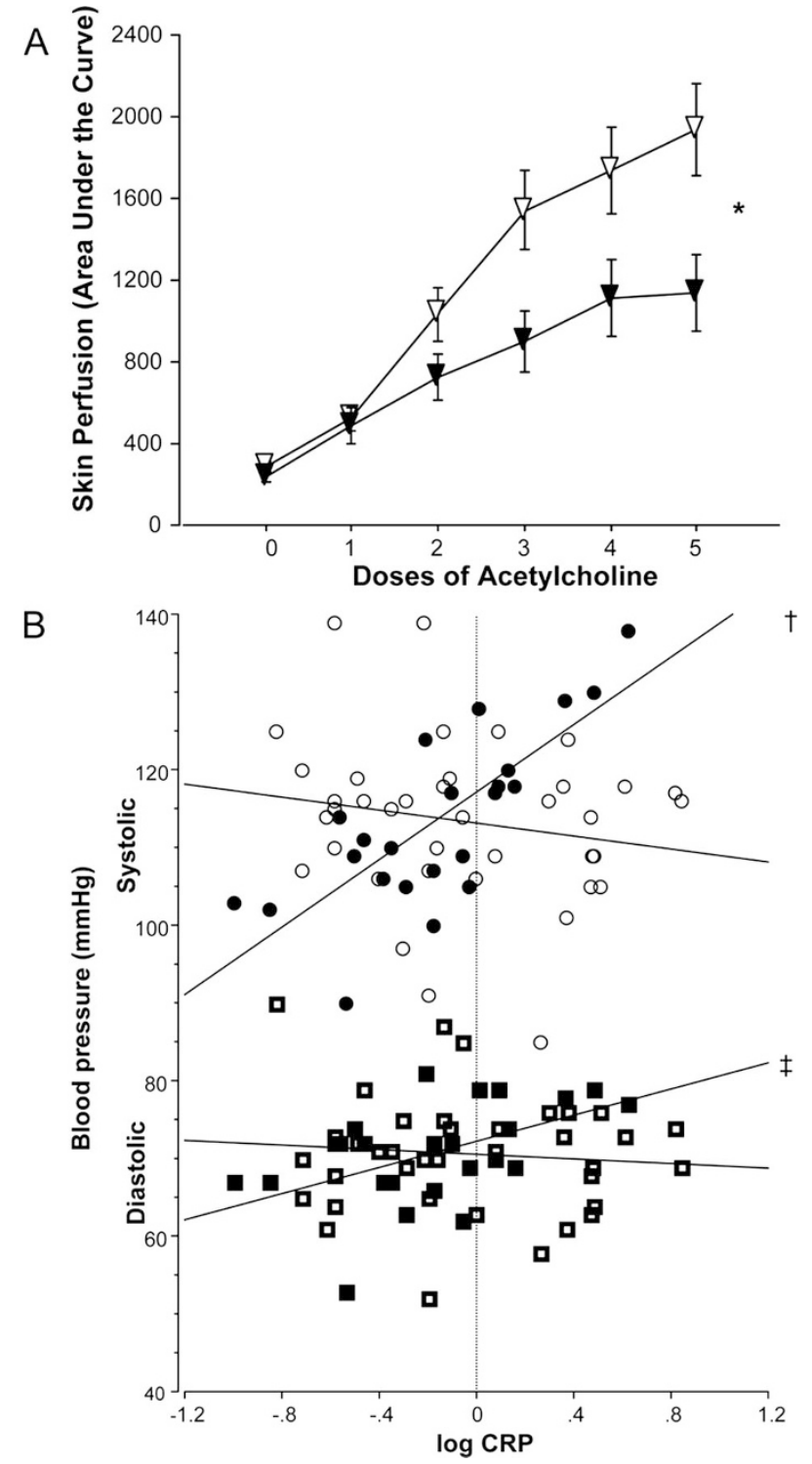

Figure 1. Skin perfusion (expressed as area under the curve) before and after cumulative doses of acetylcholine (Panel A), and the relationship between blood pressure (systolic: circles; diastolic: squares) and log CRP (Panel B) in diabetic patients with (filled symbols) and without (empty symbols) HLADQ2/8. In Panel $A$, data are expressed as mean $\pm \mathrm{SE} ; * p=0.01$. In Panel $B$, CRP is log-transformed due to its skewed distribution; $\dagger p<0.001$ and $r=$ 0.76 , and $\ddagger p=0.01$ and $r=0.50$, for systolic and diastolic blood pressure, respectively, in DQ2/8 patients.

present study is the first to link HLA-DQ2/8, which confers the highest risk for T1D, to cutaneous microvascular dysfunction in young diabetic patients. This association, which remained significant after adjustment for potential confounders, might suggest, although it does not prove, a possible role of HLA in microvascular dysfunction in T1D. This hypothesis is further supported by the significant relationship between blood pressure and CRP in DQ2/8 patients, given the contribution of microcirculation in blood pressure regulation and development of hypertension (11), and by the elevated plasma levels of sICAM-1 in these patients. ICAM-1 is a cell surface glycoprotein involved in the firm attachment of leukocytes to endothelium, and its up-regulation by cardiovascular risk factors accompanies progression of vascular disease (12). 
As endothelial cells have been shown to express HLA class II proteins, it cannot be excluded that these cells are antigenpresenting cells. Such cells are known to initiate immune responses and further studies will be needed to determine to what extent HLA class II-positive cells could contribute to endothelial cell injury.

Generalized microvascular dysfunction is an early event in patients with T1D (1) but the precise mechanisms remain unclear. Despite adequate metabolic control, patients with T1D continue to bear a significant risk of complications stemming from microcirculation (2). It is uncertain whether the risk burden could be in part related to HLA. Some, but not all, previous epidemiologic studies suggested increase in cardiovascular risk in individuals with certain HLA genotypes $(3,4,13)$. In our cohort, incipient retinopathy was detected in only few patients, without any clear difference between the DQ2/8 groups.

In one earlier study, weak correlations were found between Ach-induced microvascular responses in skin and diabetes duration or $\mathrm{HbA}_{1 \mathrm{c}}$ (14). No such associations were observed in our study but this may reside in the relatively low number of patients with poor glycemic control (i.e., 19 patients).

Two important limitations need to be considered: the study population is rather small and the cross-sectional design precludes mechanistic insights into the suggested association. Further studies are needed to validate the results and to explore the underlying mechanisms.

In conclusion, the present study suggests an association of HLA-DQ2/8 with microvascular dysfunction in juvenile T1D. Confirmation of our findings in future studies could render conceivable the hypothesis that diabetes-risk HLA may contribute to generalized microvasculopathy already before the clinical onset of diabetes. One might also speculate that this genetic influence not only contributes to future microvascular complications in T1D but could also explain the intriguing, yet significant association between HLA-DQ2/8 and T1D, given the growing concept that attributes a microvascular origin of this disease.

Acknowledgments. We thank Annica Maxedius, research nurse, for excellent technical assistance in laser Doppler and blood sampling.

\section{REFERENCES}

1. La Fontaine J, Harkless LB, Davis CE, Allen MA, Shireman PK 2006 Current concepts in diabetic microvascular dysfunction. J Am Podiatr Med Assoc 96:245252

2. Cooper ME, Bonnet F, Oldfield M, Jandeleit-Dahm K 2001 Mechanisms of diabetic vasculopathy: an overview. Am J Hypertens 14:475-486

3. Mimura T, Funatsu H, Uchigata Y, Kitano S, Noma H, Shimizu E, Konno Y, Amano S, Araie M, Yoshino O, Iwamoto Y, Hori S 2003 Relationship between human leukocyte antigen status and proliferative diabetic retinopathy in patients with younger-onset type 1 diabetes mellitus. Am J Ophthalmol 135:844-848

4. Shiina T, Inoko H, Kulski JK 2004 An update of the HLA genomic region, locus information and disease associations. Tissue Antigens 64:631-649

5. Savage CO, Brooks CJ, Harcourt GC, Picard JK, King W, Sansom DM, Wilcox N 1995 Human vascular endothelial cells process and present autoantigen to human T-cell lines. Int Immunol 7:471-479

6. Greening JE, Tree TI, Kotowicz KT, van Halteren AG, Roep BO, Klein NJ, Peakman N 2003 Processing and presentation of the islet autoantigen GAD by vascular endothelial cells promotes transmigration of autoreactive T-cells. Diabetes 52:717-725

7. Suzumura Y, Ohashi M 1993 Immunoelectron localization of HLA-DR, HLA-DP, and HLA-DQ antigens on the microvasculature in normal skin. J Am Acad Dermatol 29:202-205

8. Todd JA 1995 Genetic analysis of type 1 diabetes using whole genome approaches. Proc Natl Acad Sci USA 92:8560-8565

9. Lernmark B, Lynch K, Lernmark A 2006 Cord blood islet autoantibodies are related to stress in the mother during pregnancy. Ann N Y Acad Sci 1079:345-349

10. Morris SJ, Shore AC 1996 Skin blood flow responses to the iontophoresis of acetylcholine and sodium nitroprusside in man: possible mechanisms. J Physiol 496:531-542

11. Segal SS 2005 Regulation of blood flow in the microcirculation. Microcirculation 12:33-45

12. Hubbard AK, Rothlein R 2000 Intercellular adhesion molecule-1 (ICAM-1) expression and cell signaling cascades. Free Radic Biol Med 28:1379-1386

13. Acton RT, Bell DS, Collins J, Giger JN, Go RC, Harrison R, McDonald R, Rivers C, Roseman JM, Taylor HA, Vanichanan C 1997 Genes within and flanking the major histocompatibility region are risk factors for diabetes, insulin resistance, hypertension, and microalbuminuria in African-American women. Transplant Proc 29:3710-3712

14. Khan F, Elhadd TA, Greene SA, Belch JJ 2000 Impaired skin microvascular function in children, adolescents, and young adults with type 1 diabetes. Diabetes Care 23:215-220 